\title{
A Hierarchical Approach for Fast Calculating Minimal Cut Sets of a Microgrid
}

\author{
Yanbo Che, Yuancheng Zhao, Jianmei Xu, and Jinhuan Zhou \\ Key Laboratory of Smart Grid of Ministry of Education, Tianjin University, Tianjin 300072, China \\ Correspondence should be addressed to Yanbo Che; ybche@tju.edu.cn
}

Received 26 March 2017; Revised 23 July 2017; Accepted 20 August 2017; Published 25 September 2017

Academic Editor: David Bigaud

Copyright ( 2017 Yanbo Che et al. This is an open access article distributed under the Creative Commons Attribution License, which permits unrestricted use, distribution, and reproduction in any medium, provided the original work is properly cited.

\begin{abstract}
Minimal cut sets are the basis of reliability analysis using analytical techniques. At the present stage, minimal cut sets are mainly obtained by dealing with minimal path sets, which involves cumbersome steps and slower operational speed. The speed of reliability analysis is limited by that of calculating minimal cut sets. In consideration of the characteristics of microgrid, a hierarchical approach for fast calculating minimal cut sets is proposed in this paper. Firstly, an equivalent principle is proposed to convert topology structure into network node diagram; then grades of nodes are designated based on their original connection and the breadth-first search approach; afterwards, root-leaf matrices and selected matrix are created to specify the direction and order of the search. Next, all possible combinations of minimal cut sets are vertically traversed out by replacing root nodes with leaf nodes to achieve a more rapid access to minimal cut sets. Finally, taking the electrical structure of type A380 more electric aircraft as an example, mainstream methods are compared to show the correctness and advantage of our proposed method.
\end{abstract}

\section{Introduction}

A microgrid is a small electrical power generation and distribution system consisting integrally of distributed generation, loads, energy storage devices, converters, monitoring, and protection devices [1-3]. Reliability, accessibility, and flexibility of microgrids have become hot spots of research at home and abroad.

Compared to the microgrid, the traditional grid is mainly characterized with high safety margin and strong selfhealing, so it can be regarded as a repairable system. Based on the statistical data or empirical data [4], the reliability analysis of traditional grid is using Monte Carlo simulation method $[5,6]$ and Markov algorithm [7] centered analytic method.

The approach proposed in this paper focuses on isolated microgrid. The isolated microgrid will have lower safety margin and antirisk ability when it loses the support of the traditional grid. Considering the actual operation, many faults cannot be eliminated in time, and the isolated microgrid inclines to be an unrepairable system in essence. Therefore, the minimal cut sets are used as core in the reliability analysis method of the unrepairable system. The calculation of minimal cut sets directly restricts the speed and efficiency of reliability analysis, and a lot of works have been done in the face of bottleneck problem.

Reference [8] presents a measure based on minimal cut sets to evaluate the reliability of the whole system. Minimal path sets are obtained by incidence-matrix method and then inverted to minimal cut sets in $[9,10]$. Literature [11] summarizes the applications of minimal cut sets and proposes a new algorithm for minimal cut sets based on correlation with direct partial Boolean. Literature [12] proposes a new algorithm based on Boolean satisfiability problem to solve the minimal cut sets in fault tree analysis. Application of Boolean logic system is a new trend of identifying minimal cut sets. Literature [13] presents an approach that the DepthFirst search is applied to generate minimal cut sets. Literature [14] presents an algorithm for minimal cut set identification of coherent and noncoherent Faults Trees. Reference [15] proposes a new method for minimal cut sets generation of coherent Faults Trees without repeated events. Reference [16] proposes a new formulation capable of correctly identifying the minimal cut sets needed. Because of the wide application of Faults Trees, almost literatures take those as the research object to obtain the minimal cut sets and simplify complex system. Nevertheless, few attentions have been paid to the 


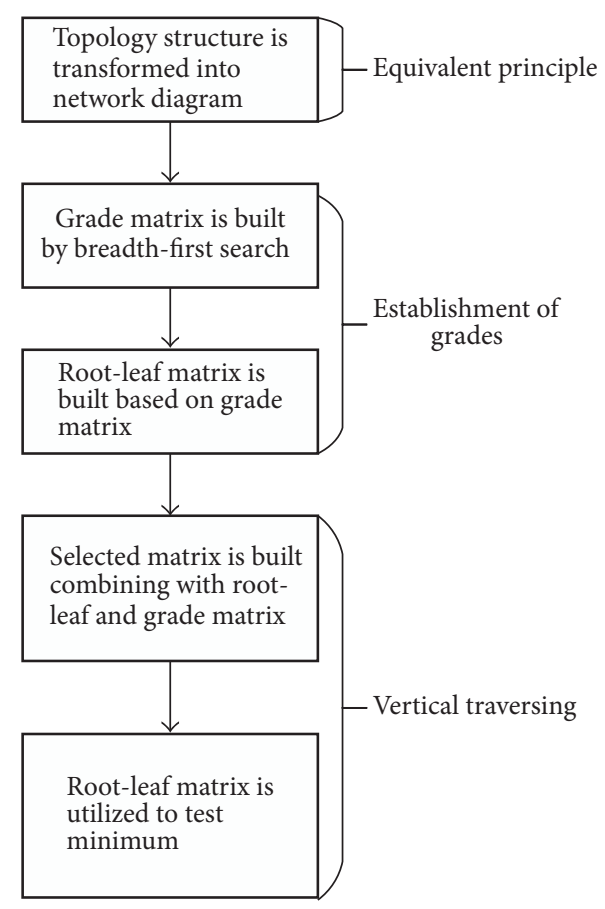

FIGURE 1: The overall flow diagram of the hierarchical approach.

way of getting minimal cut sets in graph theory and improvement of speed.

There are three ways to get the minimal cut sets nowadays [17]: by inverting minimal path sets according to De Morgan's laws; by OR operation of minimal path sets for minimal cut sets; by the method of imitative dual graph. Involving tedious steps and low efficiency, those ways cannot obtain minimal cut sets directly from network nodes diagram.

In consideration of the characteristics of unrepairable and simple structure in the microgrid, this paper presents a quick way to calculate minimal cut sets, that is, hierarchical approach. First of all, an applicable equivalent principle is proposed that convert structure of power system into network node diagram based on graph theory. Nodes are classified and ranked by breadth-first search; then the total minimal cut sets are traversed out by replacing high graded nodes with low graded nodes. Taking the electrical power system in type A380 more electric aircraft as an example, this paper verifies the correctness and superiority of the proposed method by comparisons.

\section{Hierarchical Approach}

Different from the traditional method from minimal path sets to minimal cut sets, the hierarchical approach designates grades for nodes; then all nodes are traversed in terms of their grades vertically to get the minimal cut sets. The overall flow diagram of the hierarchical approach is shown in Figure 1, which includes topology equivalence, establishment of grades, and vertical traversing.

2.1. Topology Equivalence. In this step, topology structure is transformed into network node diagram. Generally speaking, for a large grid, the follow guidelines must be obeyed: the network node diagram should reflect connections and working status of the original topology structure; the network node diagram should be as simple and easy as possible to enable making full use of the functions of computers.

However, there are many differences between microgrid and traditional grid. As electrical components in a microgrid feature with unrepairable, simple structure, and low concurrency, it is possible to find a more proper and simple equivalent principle when converting topology structure into network node diagram by considering such specifications. Hence, the concrete rules adopted are as follows:

(1) All components, such as generator, transformer rectifier, and DC-bus, are replaced by nodes.

(2) Arcs between nodes only reflect their connection relationship and direction of power flow. Any arc connecting a node standing for a component controlling energy flow is directed arc, such as transformer rectifiers and diodes; otherwise, it is undirected.

(3) Any single-line bidirection connected node is permitted to be absorbed by other nodes jointed with multiline or by directed arcs.

(4) Set a virtual node as source of power injected into the entire microgrid. Power is injected directly to nodes standing for generators or batteries from virtual source. Load nodes are taken as trap nodes consuming all power energy, and their arcs do not consume any power.

Different from the mainstream equivalent rules, this principle has two particular improvements combining with features of microgrid:

(1) All components in microgrid are represented as nodes because of their simple connection relationship, and an arc just represents a relationship existing between two nodes. So, it needs not to classify and discuss nodes and arcs.

(2) Only critical nodes stand for the reliability of an overall system, and irrelevant nodes are allowed to be extensively absorbed. Nodes outside research range or with unidirectional flow of energy can be replaced with relevant nodes surrounding since they have few impact on critical nodes. This rule can greatly reduce the total number of nodes in the system and increase operation speed.

2.2. Establishment of Grades. This procedure is to designate grades for nodes in a network node diagram by considering the directions of energy flow using breadth-first search and then to build root-leaf matrix based on the grades of nodes. For the convenience of statement, relationship between nodes with the same grade is entitled "horizontal," while relationship between nodes with different grades is entitled "vertical."

This section mainly includes how to create grade matrix and root-leaf matrix. 


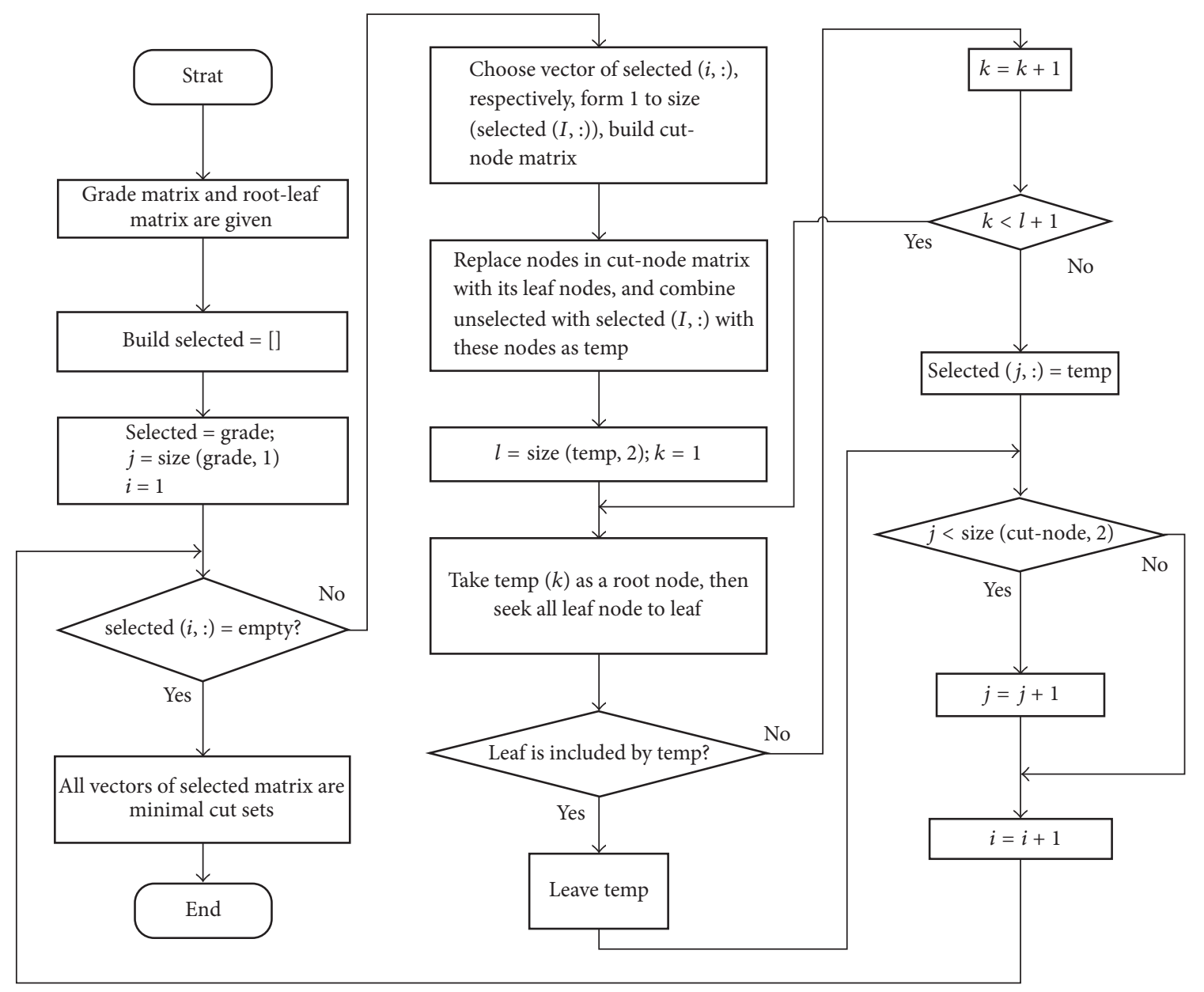

FIGURE 2: Flowchat for obtaining minimal cut sets on the basis of root-leaf matrix.

2.2.1. Building Up Grade Matrix. Grades of nodes are designated by breadth-first search: the length of an arc is recorded as 1 . For a specific node, its distance to the source node equals the number of arcs between them, which is defined as its grade. In this way, all nodes in network node diagram have their own grades $(1,2, \ldots, n-1)$. If a node has two grades, then the lower grade is selected. A node with smaller grade means a node of higher level. Then grade matrix of nodes is formulated according to their grades, of which row vectors stand for grades and column vectors stand for numbers of nodes.

The source node is a virtual node which grade is set as 0 . Grades of nodes connected with directed arcs are adjusted in this way that a directed arc is pointed from node at high grade (small value) to node at low grade. The nodes with the same grades can be connected by undirected arcs to indicate that flow energy is undirected.

2.2.2. Building Up Root-Leaf Matrix. Row vectors of a rootleaf matrix stand for leaf nodes and column vectors of that stand for root nodes. There are two numbers 0 and 1 in this matrix, in which 1 stands for existence of the root node and the leaf node, while 0 stands for nonexistence of those. For two directly connected nodes, the one at high grade is taken as root of the other one, while the one at low grade is taken as leaf of the other one.

Obviously, a leaf node could be root of nodes at lower level. On the one hand, the root-leaf matrix could be used as a basis for vertical traversing and, on the other hand, as a tool for testing minimum.

2.3. Vertical Traversing. Vertical traversing is to search for all the combination of all nodes. According to grades of nodes, root nodes are replaced with leaf nodes in vertical direction so as to find all free combination among different grades. To prevent that different root nodes have the same leaf node, minimized testing must be performed to ensure all minimal cut sets obtained are different.

Figure 2 shows the block diagram of the algorithm in this section, the core of the approach. The row vector of the selected matrix stands for number of nodes and the column vector of that stands for all possible cut sets which could become minimal cut sets.

The variable $i$ is the maximum number of row vectors of selected matrix. The variable $j$ is the number of column vectors of selected matrix, which equals the total number of nodes. The variable $k$ is the number of nodes in the vector "temp" extracted from selected matrix and helps search all 


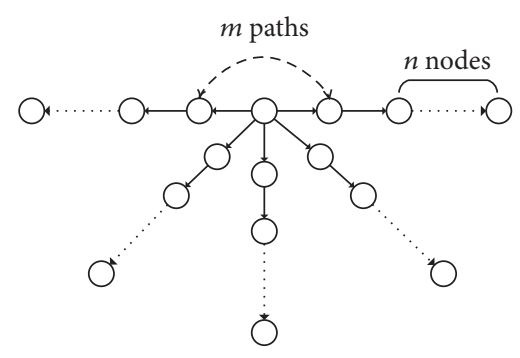

Figure 3: A radial network diagram.

nodes, while the variable $l(l<i)$ represents a specific row in selected matrix and helps to select all row vectors.

2.3.1. Building Up Selected Matrix. All possible cut sets are stored in the selected matrix, in which each row vector represents one cut set. The selected matrix is initialized as the grade matrix. A row vector is extracted from selected matrix, and $1,2, \ldots, k-1$ nodes are extracted from this row vector, respectively, in sequence. A new vector is formed, by replacing the extracted nodes with their leaf nodes and combining them with the nodes unselected in this vector.

This new cut set will be put into the selected matrix as a new vector once it is confirmed to be different from those existing in the selected matrix and passes test of minimization. This process is repeated until no new cut set is generated and all vectors in selected matrix have been analyzed. The left in selected matrix gives all minimal cut sets.

2.3.2. Testing Minimization. It is said that a newly formed vector is minimized if leaf nodes of any arbitrary node are not completely contained by its previous vector. This method of testing minimization is only applicable for a new vector obtained each time.

\section{Advantage Analysis}

Figure 3 is a radial network diagram with $m$ paths, each path having $n$ nodes. Taking Figure 3 as an object, it is proved by mathematical analysis that the proposed approach is superior to mainstream in terms of calculation speed. If the structure is more complex, the calculation speed of approach proposed is faster in theory. Because the hierarchical approach sets grades that can describe the direction of energy flow, and it is avoided that the direction of search is inconformity with energy flow obviously. Traversal in mainstream method leads to an increase in calculation and a decrease in speed.

The standard of comparison is the time required to complete all the calculation. It is supposed that a basic operation (it refers to a logical operation or search for a node) costs $t$ on average.

The time of mainstream method is $T_{\text {ori }}$, and calculation is shown in

$$
T_{\text {ori }}=T_{\text {ori1 }}+T_{\text {ori2 }} \text {. }
$$

It mainly consists of two parts: the minimal path set is obtained by depth-first search, costing $T_{\text {oril }}$; the minimal cut set is obtained by operation "OR" on minimal path set, costing $T_{\text {ori2 }}$. Each path costs $n t$ for searching from the source node to targeted node. It costs $n t$ that source node is tracked back after searching a path. The total time for searching $m$ paths is shown in

$$
T_{\text {ori1 }}=2 m n t .
$$

The first-order minimal cut set can be directly obtained, and the second-order minimal cut set is obtained by operation "OR" on nodes corresponding to any two paths. Similarly, third, fourth, and $m$ order cut set can be obtained. The time of second part is shown as

$$
T_{\text {ori2 }}=\left(C_{m}^{2}+C_{m}^{3}+\cdots+C_{m}^{m}\right) \times n t .
$$

Equation (3) is simplified to (5), shown as follows based on (4):

$$
\begin{aligned}
C_{n}^{0}+C_{n}^{1}+C_{n}^{2}+\cdots+C_{n}^{n} & =2^{n}, \\
T_{\text {ori } 2} & =\left(2^{m}-m-1\right) \times n t .
\end{aligned}
$$

The proposed approach costs $T_{\text {hie }}$, as shown in

$$
T_{\text {hie }}=T_{\text {hie1 }}+T_{\text {hie2 }} .
$$

The hierarchical approach includes two parts: set up grades by using breadth-first search, costing $T_{\text {hie }}$; all nodes are traversed vertically, costing $T_{\text {hie }}$. There are $n$ grades and a grade is set up by costing $2 m t$, so $T_{\text {hiel }}=2 m n t$. All cut sets are traversed, costing $m n t$, by replacing the root nodes from leaf nodes on every grade. It costs mnt to test minimization of all cut sets $T_{\text {hie } 2}=2 m n t$.

The time taken by the two methods is compared, as shown in

$$
T_{\text {ori }}-T_{\text {hie }}=\left(2^{m}-(3 m+1)\right) n t .
$$

The mainstream method needs more time than the hierarchical approach, when $m>3$. It estimated conservatively that the above conclusion can be drawn. In actual microgrid, every node has more redundancy path for power supply and the paths must be more complex. The amount of search paths increases exponentially as the number of nodes increases in mainstream method. The amount of search is limited based on energy flow in hierarchical approach, so it is proved that the approach proposed can save calculation time and speed up operation in mathematical theory.

\section{Case Study}

Electrical power systems of more electric aircraft are characterized by unrepairable, simple connection, low concurrency, and high redundancy. In consideration of these characters and the topologies, they can be regarded as typical isolated microgrids. The electrical power system of Airbus 380, a representative of more electric aircraft, is taken as a case to test the approach presented, whose electrical structures are shown in Figure 4.

The overall electrical system is roughly divided into four main channels: E1 channel is powered by AC generators G1 


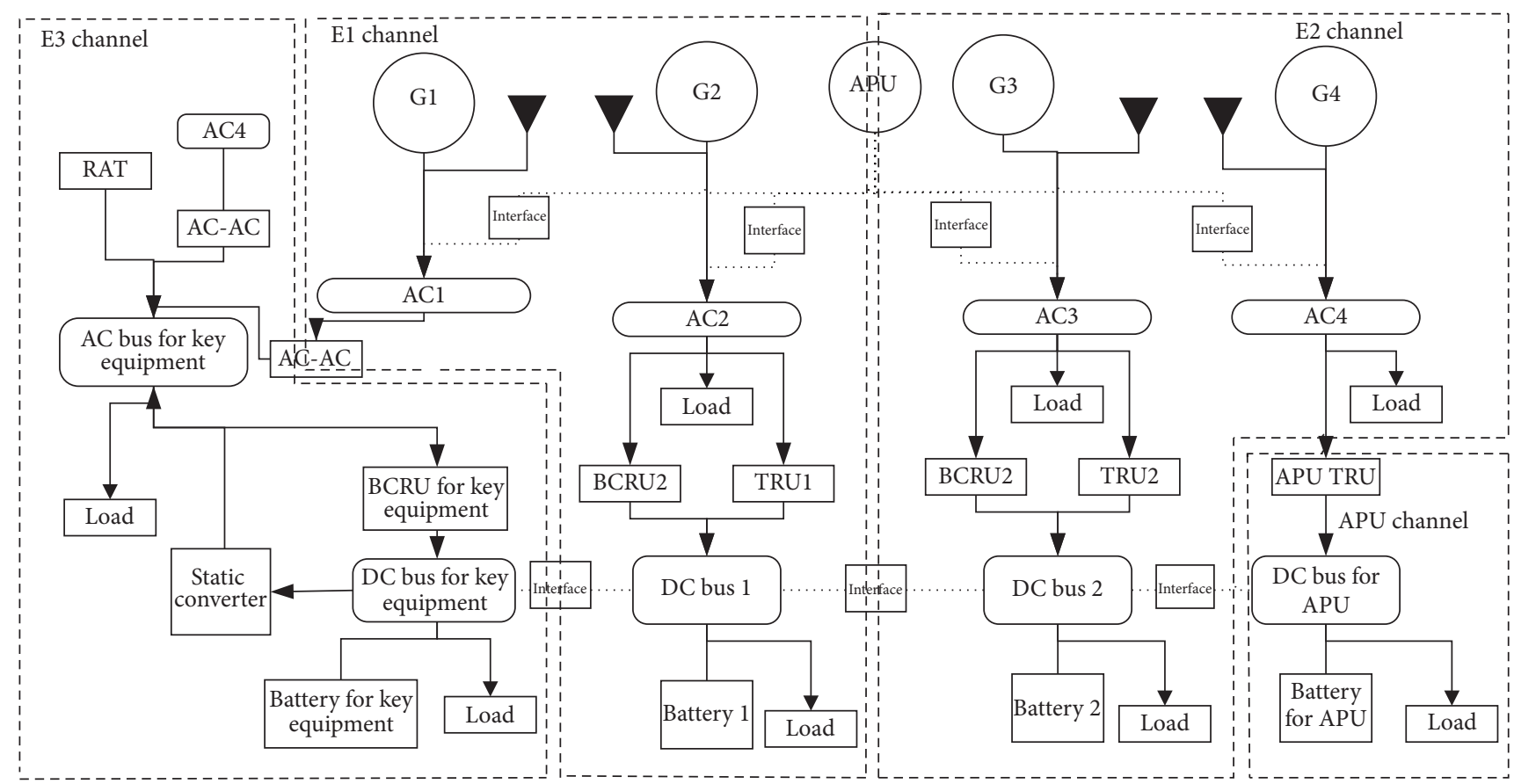

Figure 4: Power system structure sketch of A380.

and G2; E2 channel by AC generators G3 and G4; E3 channel by RAT (Ram Air Turbine) and static converters; and APU (Auxiliary Power Unit) channel associated with APU startup.

A380 utilizes variable frequency generating technology, so the AC-buses operate independently, while the DC-buses run in parallel. This parallel-cluster combines the advantages of operation of independent and parallel: on the one side, the DC system in parallel could ensure uninterrupted power supply; on the other hand, independent operation could effectively prevent the occurrences of cascading failure.

4.1. Equivalent Transformation. According to the equivalent principle presented in Section 2.1, the structure in Figure 4 is transformed into the network node diagram shown in Figure 5.

The power is generated from the virtual source node v1 and the flows into AC-buses (v8, v9, v10, and v11) through generators (v3, v4, v6, and v7). Node v2 represents that power is generated from RAT into AC-buses for keeping the key equipment running on emergency. Node v5 represents APU. Node v20 represents AC-bus for key equipment, while node v21 represents static converter or BCRU, depending on the direction of energy flow. Power electronics interface, representation of nodes $\mathrm{v} 13, \mathrm{v} 14,15, \mathrm{v} 16, \mathrm{v} 25, \mathrm{v} 27$, and $\mathrm{v} 29$, is used to connect different buses. DC-buses are represented by nodes v24, v26, v28, and v30.

As mentioned in the equivalent principle, a portion of irrelevant loads can be absorbed by power supply nodes nearby. For example, the AC loads are absorbed by nodes 9 , 10 , and 11 represented by AC-bus.

4.2. Analyzed Procedures. As indicated in Section 2.2, nodes are classified into different grades by breadth-first search,
TABLE 1: Entire minimal cut sets.

\begin{tabular}{lc}
\hline & Node 20 \\
\hline 1 & $\mathrm{v} 20$ \\
2 & v8v1lv24v2 \\
3 & v8v1lv26v2 \\
4 & v8v1lv21v2 \\
5 & v8v1lv25v2 \\
6 & $\mathrm{v} 8 \mathrm{v} 24 \mathrm{v} 2 \mathrm{v} 22$ \\
7 & $\mathrm{v} 8 \mathrm{v} 26 \mathrm{v} 2 \mathrm{v} 22$ \\
8 & $\mathrm{v} 8 \mathrm{v} 2 \mathrm{v} 22 \mathrm{v} 21$ \\
9 & $\mathrm{v} 8 \mathrm{v} 2 \mathrm{v} 22 \mathrm{v} 25$ \\
10 & $\mathrm{v} 11 \mathrm{v} 24 \mathrm{v} 2 \mathrm{v} 12$ \\
11 & $\mathrm{v} 11 \mathrm{v} 26 \mathrm{v} 2 \mathrm{v} 12$ \\
12 & $\mathrm{v} 11 \mathrm{v} 2 \mathrm{v} 12 \mathrm{v} 21$ \\
13 & $\mathrm{v} 11 \mathrm{v} 2 \mathrm{v} 12 \mathrm{v} 25$ \\
14 & $\mathrm{v} 24 \mathrm{v} 2 \mathrm{v} 12 \mathrm{v} 22$ \\
15 & $\mathrm{v} 26 \mathrm{v} 2 \mathrm{v} 12 \mathrm{v} 22$ \\
16 & $\mathrm{v} 2 \mathrm{v} 12 \mathrm{v} 22 \mathrm{v} 21$ \\
17 & $\mathrm{v} 212 \mathrm{v} 22 \mathrm{v} 25$ \\
\hline
\end{tabular}

which are the basis of searching in vertical direction. The network node diagram with grades is obtained from Figure 5 by breadth-first search, shown in Figure 6, in which the number boxed near a node stands for its grade.

As indicated in Section 2.3, the entire minimal cut sets are obtained, shown in Table 1, based on vertically traversing root-leaf matrix. If all components involved in any minimal cut set fail at the same time, the appointed node would lose power, and even the topology reconfiguration could not help. 




FIgURE 5: Network nodes diagram.

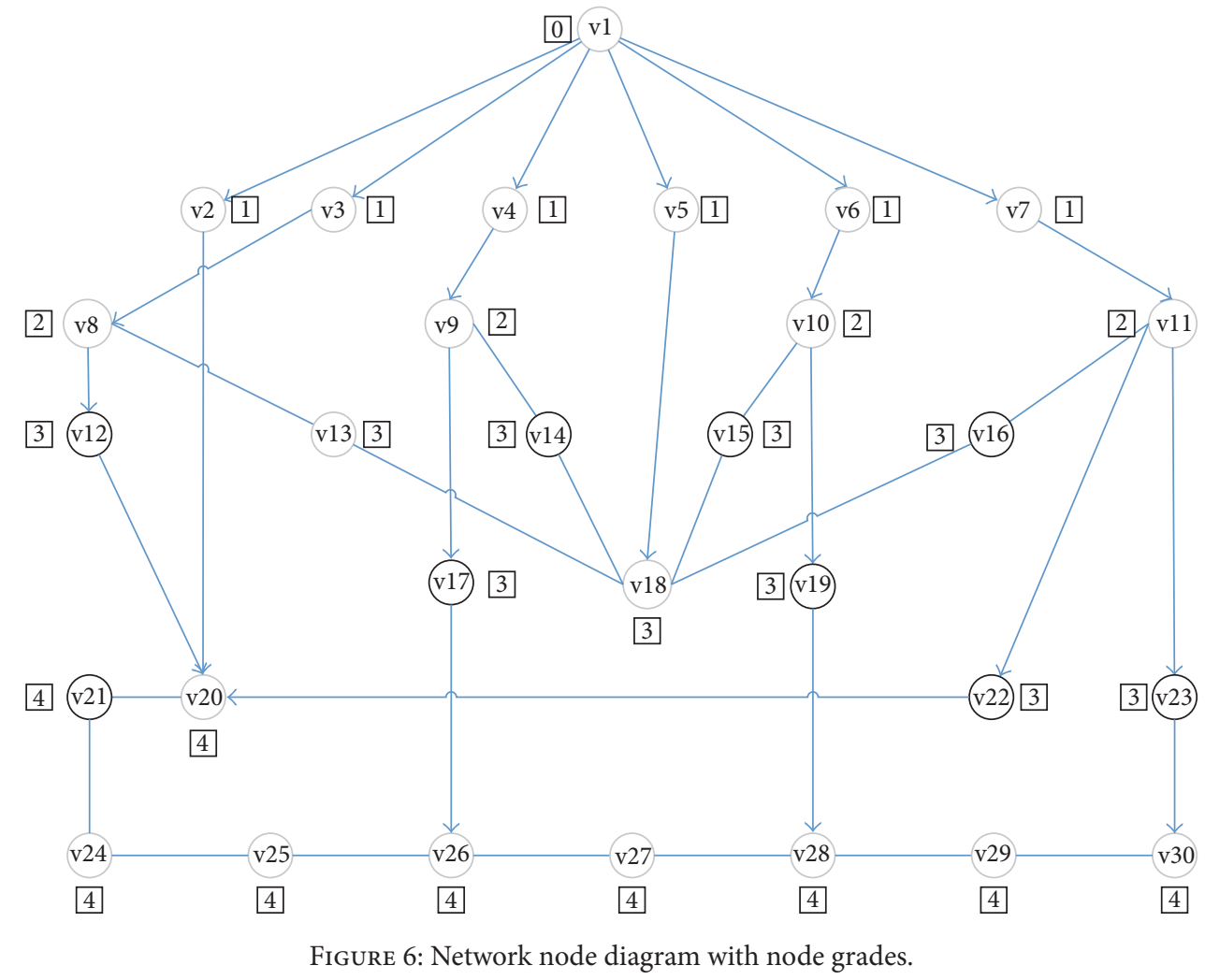


TABLE 2: Minimal path sets from source to node 20.

\begin{tabular}{|c|c|}
\hline Length of path & Node 20 \\
\hline 3 & vlv2v20 \\
\hline \multirow{2}{*}{5} & vlv3v8v12v20 \\
\hline & vlv7v1lv22v20 \\
\hline \multirow{2}{*}{7} & vlv5v18v13v8v12v20 \\
\hline & vlv5v18v16v1lv22v20 \\
\hline \multirow{7}{*}{9} & vlv4v9v14v18v13v8v12v20 \\
\hline & vlv6v10v15v18v13v8v12v20 \\
\hline & vlvv7v11v16v18v13v8v12v20 \\
\hline & vlv3v8v13v18v16v11v22v20 \\
\hline & vlv4v9v14v18v16v11v22v20 \\
\hline & v1v6v10v15v18v16v11v22v20 \\
\hline & v1v4v9v17v26v25v24v21v20 \\
\hline \multirow{2}{*}{11} & vlv5v18v14v9v17v26v25v24v21v20 \\
\hline & v1v6v10v19v28v27v26v25v24v21v20 \\
\hline \multirow{5}{*}{13} & v1v3v8v13v18v14v9v17v26v25v24v21v20 \\
\hline & v1v6v10v15v18v14v9v17v26v25v24v21v20 \\
\hline & vlv7v11v16v18v14v9v17v26v25v24v21v20 \\
\hline & v1v5v18v15v10v19v28v27v26v25v24v21v20 \\
\hline & v1v7v11v23v30v29v28v27v26v25v24v21v20 \\
\hline \multirow{4}{*}{15} & v1v3v8v13v18v15v10v19v28v27v26v25v24v21v20 \\
\hline & vlv4v9v14v18v15v10v19v28v27v26v25v24v21v20 \\
\hline & v1v7v11v16v18v15v10v19v28v27v26v25v24v21v20 \\
\hline & v1v5v18v16v11v23v30v29v28v27v26v25v24v21v20 \\
\hline \multirow{3}{*}{17} & vlv3v8v13v18v16v11v23v30v29v28v27v26v25v24v21v20 \\
\hline & vlv4v9v14v18v16v11v23v30v29v28v27v26v25v24v21v20 \\
\hline & vlv6v10v15v18v16v11v23v30v29v28v27v26v25v24v21v20 \\
\hline
\end{tabular}

4.3. Contrast Effects. Power system of more electric aircraft is taken as an example to be equivalent to network node diagram with 30 nodes. The approach is in comparison with mainstream on platform of MATLAB about operational time and result. There is the same result between two methods. Except the artificial step of equivalence, minimal cut sets are got after calculating minimal path sets detailed in Table 2 in the mainstream method, which cost 5.042 seconds, while this proposed approach just costs 1.389 seconds to obtain the same result, which was 3.653 seconds ahead of mainstream.

The superiorities of this method were verified in the field of a microgrid, and this method would have a faster speed and wider application on structure with more nodes.

\section{Conclusion}

Minimal cut sets play important roles in reliability assessment. This paper presents a hierarchical approach for fast calculating minimal cut sets of a microgird. This approach simplifies the steps to get minimal cut sets and increases operation speed. Quick calculation of minimal cut sets can improve the speed of reliability analysis; it also can reduce memory footprint during the procedure of reliability analysis by avoiding minimal path sets.

A topological equivalence principle for microgrid is also proposed, which potentially advances the pace of study on reliability assessment. Meanwhile, the approach proposed broadens applications of the breadth-first search and enhances the feasibility of reliability assessment based on minimal cut sets. This approach can also be extended and applied to all forms of microgrids, which is provided as a supplementary method to the existing reliability assessment system.

\section{Conflicts of Interest}

The authors declare that they have no conflicts of interest.

\section{Acknowledgments}

The authors would like to acknowledge financial support from National Natural Science Foundation of China (U1533126).

\section{References}

[1] Y. Xinfa, S. Jian, and L. Zhiping, "Overview on micro-gird technology," in Proceedings of the CSEE, pp. 57-70, china, 2014.

[2] W. Chengshan, Y. Zhangang, W. Shouxing, and C. Yanbo, "Analysis of structural characteristics and control approaches of experimental microgird systems," Automation of Electric Power Systems, vol. 34, no. 1, pp. 99-105, 2010. 
[3] N. Hatziargyriou, H. Asano, R. Iravani, and C. Marnay, "Microgrids: an overview of ongoing research, development, and demonstration projects," IEEE Power and Energy Magazine, vol. 5, no. 4, pp. 78-94, 2007.

[4] S. Ramabhotla, S. Bayne, and M. Giesselmann, "Reliability optimization using fault tree analysis in the grid connected mode of microgrid," in Proceedings of the 8th Annual IEEE Green Technologies Conference, GreenTech 2016, pp. 136-141, Kansas City, MO, USA, April 2016.

[5] R. Billinton and W. Li, Reliability Assessment of Electric Power Systems Using Monte Carlo Methods, Springer US, Boston, Mass, USA, 1994.

[6] R. Billinton and A. Jonnavithula, "Application of sequential Monte Carlo simulation to evaluation of distributions of composite system indices," IEE Proceedings - Generation, Transmission and Distribution, vol. 144, no. 2, pp. 87-90, 1997.

[7] G. Yongji, Principle of Reliability Engineering, Tsinghua University press, Beijing, China, 2002.

[8] Z. Ling, Y. Shanshui, and etal., "Reliability assessment and analysis of large aircraft power distribution systems," Acta Aeronaution et Astronautica Sinica, vol. 32, no. 8, pp. 1488-1496, 2011.

[9] L. Zongxiang and G. yongji, "Reliability evaluation of hydroelectric power station bus systems arrangement," Automation of Electric Power Systems, vol. 18, pp. 16-19, 2001.

[10] L. Hongjiang, L. Zongxiang, W. Miao, and etal., "Contrasting analysis of shipboard power system topological structures based on reliability model," Transactions of China Electrotechnical Society, vol. 21, no. 11, pp. 47-53, 2006.

[11] M. Kvassay, V. Levashenko, and E. Zaitseva, "Analysis of minimal cut and path sets based on direct partial Boolean derivatives," Journal of Risk and Reliability, vol. 230, 2016.

[12] L. Wei-lin, W. Ou, and H. Ming-yu, "Computing minimal cut sets of fault tree using SAT solver," Computer Engineering and Science, vol. 39, no. 4, pp. 725-733, 2017.

[13] Z. Miao, R. Niu, T. Tang, and J. Liu, "A new generation algorithm of fault tree minimal cut sets and its application in CBTC system," in Proceedings of the 2013 IEEE International Conference on Intelligent Rail Transportation, IEEE (ICIRT '13), pp. 221-226, Beijing, China, September 2013.

[14] F. Di Maio, S. Baronchelli, and E. Zio, "Hierarchical differential evolution for minimal cut sets identification: application to nuclear safety systems," European Journal of Operational Research, vol. 238, no. 2, pp. 645-652, 2014.

[15] D. MakajićNikolić, M. Vujošević, and N. Nikolić, "Minimal cut sets of a coherent fault tree generation using reverse Petri nets," Optimization, vol. 62, no. 8, pp. 1069-1087, 2013.

[16] L. Tobalina, J. Pey, and F. J. Planes, "Direct calculation of minimal cut sets involving a specific reaction knock-out," Bioinformatics, vol. 32, no. 13, pp. 2001-2007, 2016.

[17] G. Zhizhong, Electric Power Network Analysis, Science Press, Beijing, China, 2008. 


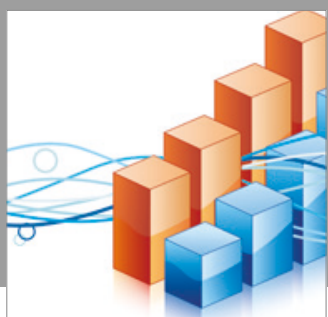

Advances in

Operations Research

vatersals

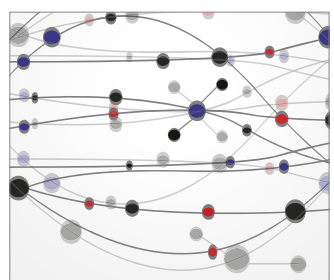

\section{The Scientific} World Journal
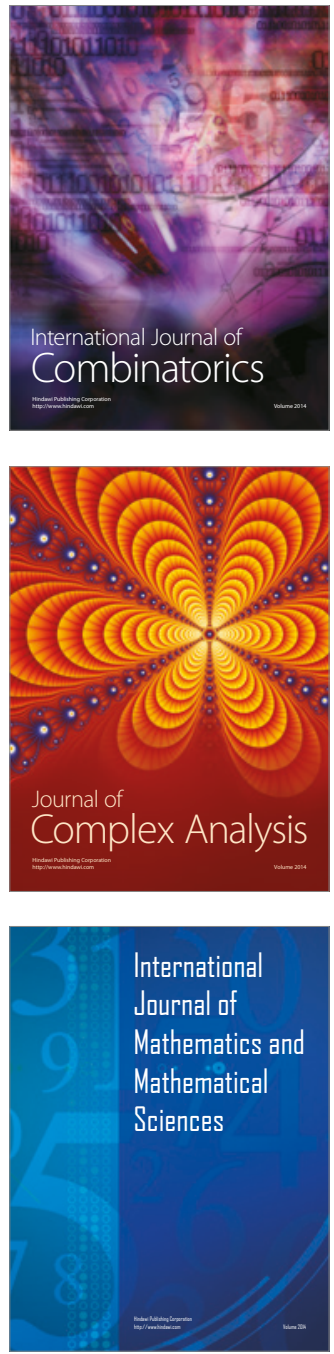
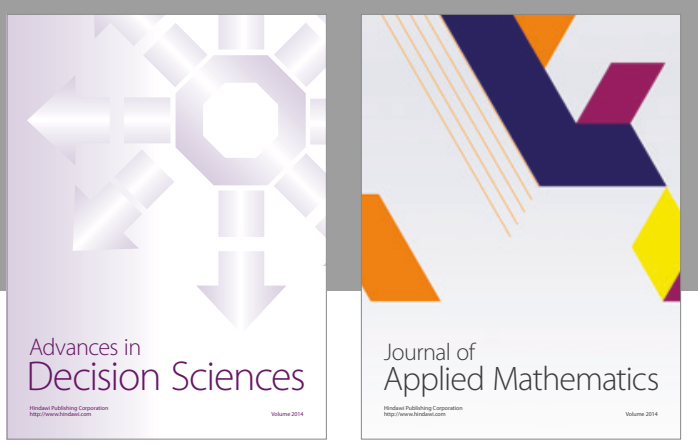

Algebra

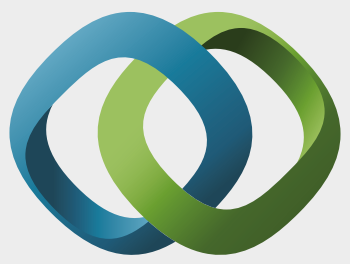

\section{Hindawi}

Submit your manuscripts at

https://www.hindawi.com
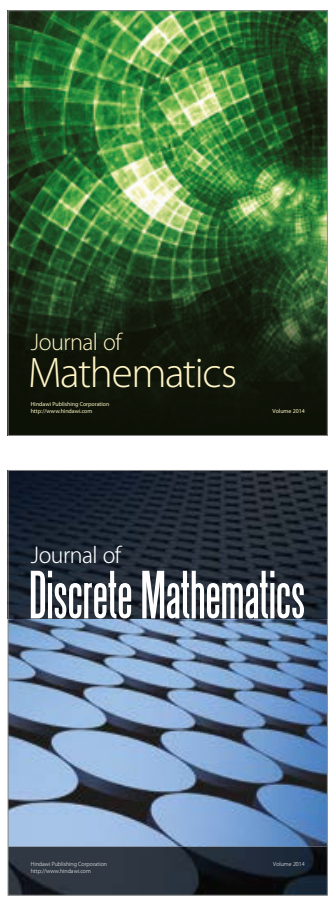

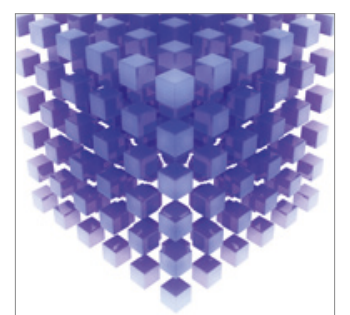

Mathematical Problems in Engineering
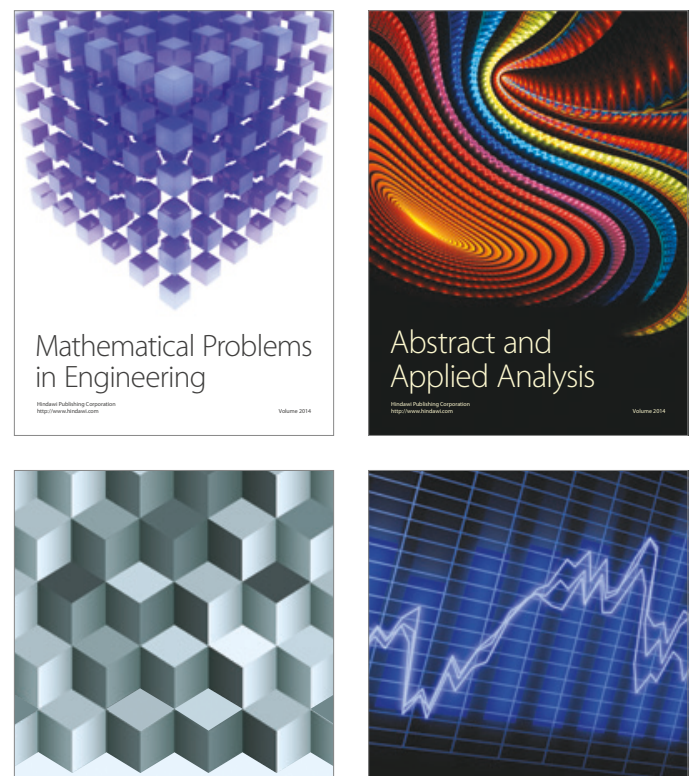

Journal of

Function Spaces

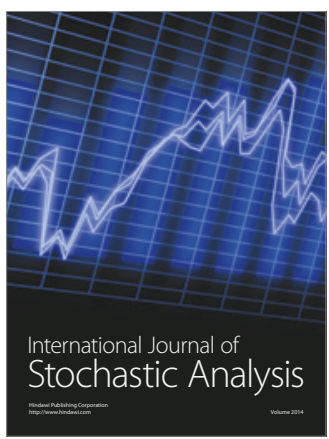

Probability and Statistics
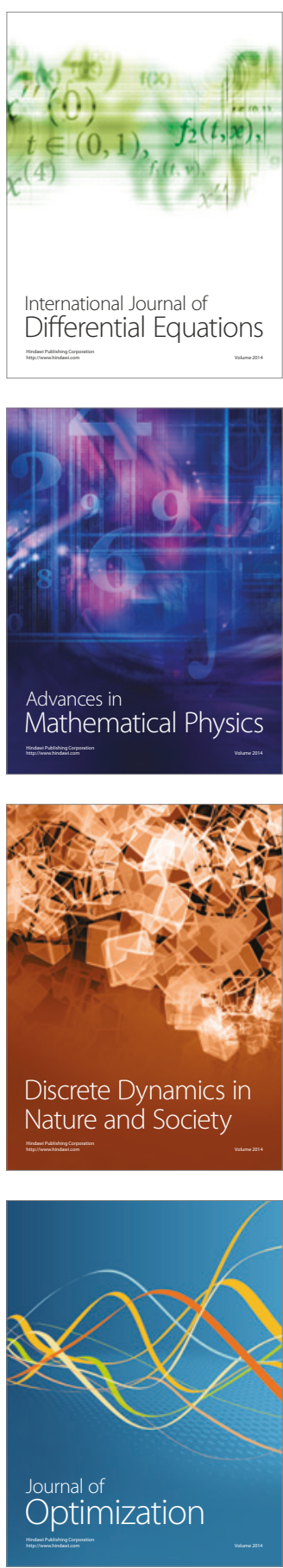